\title{
Unicystic ameloblastoma- A case report
}

\author{
Shaik Ali Hassan ${ }^{1 *}$, Sumit Bhateja ${ }^{2}$ \\ ${ }^{1}$ Dental Surgeon, ${ }^{2} \mathrm{HOD}$, Manav Rachna Dental College, Faridabad, Haryana, India \\ *Corresponding Author: Shaik Ali Hassan \\ Email: alishaikhassan@gmail.com
}

\begin{abstract}
It is the most common benign tumor that can be seen significantly. It is common odontogenic tumor involving the molar ramus area. As this tumor shows some similarities with dentigerous cysts, both clinically and radiographically the biologic behaviour of this tumor group was reviewed. The recurrence is more in unicystic ameloblastoma and patient has to have the long time followup. Here we are presenting a case of unicystic ameloblastoma in 55year female who has got edentulous ridge.
\end{abstract}

Keywords: Rests of dental lamina, From a developing enamel organ, From the epithelial lining of an odontogenic cyst, Or from the basal cells of the oral mucosa.

\section{Introduction}

Ameloblastomas are benign tumors as its importance lies in its potential to grow into enormous size with resulting bone deformity. They are typically classified as unicystic, multicystic, peripheral and malignant subtypes. ${ }^{1}$ Ameloblastomas are tumors of odontogenic epithelial origin. Theoretically, they may arise from rests of dental lamina, from a developing enamel organ, from the epithelial lining of an odontogenic cyst, or from the basal cells of the oral mucosa. Unicistic ameloblastoma is a less common variant of ameloblastoma, which refers to cystic lesions having clinical and radiographic signs of the odontogenic cyst, but histological examination reveals a typical ameloblastomatous epithelial lining of the cyst cavity with or without tumor proliferation. $^{2}$ Ameloblastomas are slow-growing, locally invasive tumors that have a benign course in most cases.

\section{Case Report}

A 55 year old female patient came to the department of oral medicine and radiology for the chief complaint of swelling in right posterior back tooth region and increasing in size, since 2 to 3months. Patient doesn't have any history of smoking, alcohol, betel nut. There was no associated pain, patient didn't had any difficulty in opening mouth, chewing. The patient's past medical history was normal, the patient was asymptomatic and there was no associated mandibular or intraoral swelling or lymphadenopathy of the head and neck.

On extraoral examination there was a swelling of size $5.0 \times 3.0 \times 2.0 \mathrm{~cm}$, which extended from corner of lip to the angle of mandible. An extraoral radiograph that is orthopantogram (OPG) was taken which revealed the osteolytic lesion measuring approximately $5.0 \times 3.0 \times 2.0 \mathrm{~cm}$, localized at the right retromolar area distal to the lower right first molar region and pushing the $3^{\text {rd }}$ molar to distally. On clinical examination revealed a vestibular swelling in region of molar, non erytmatous, non tender.

Fnac was performed and fluid was taken from the swelling and after that it was stained which reveaed that the fi brous cyst wall with a lining that consists totally or partially of ameloblastic epithelium. It demonstrates a basal layer of columnar or cuboidal cells with hyperchromatic nuclei that show reverse polarity and basilar cytoplasmic vacuolization. The overlying epithelial cells are loosely cohesive and resemble stellate reticulum.

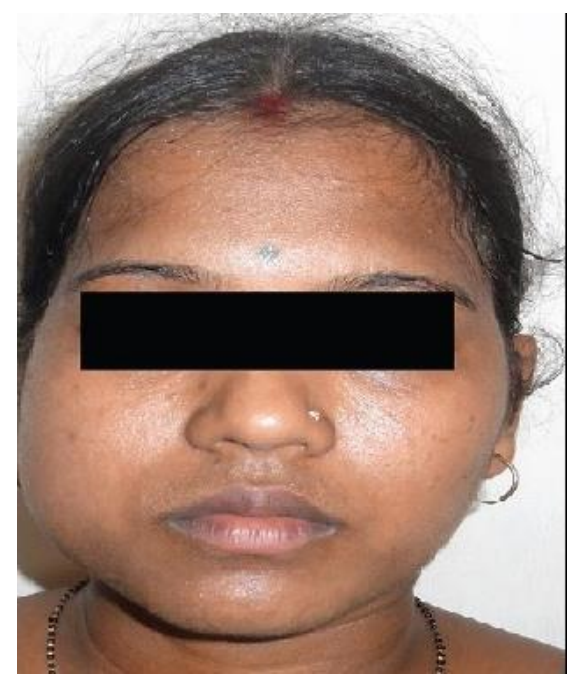

Fig. 1: Extraoral picture of patient

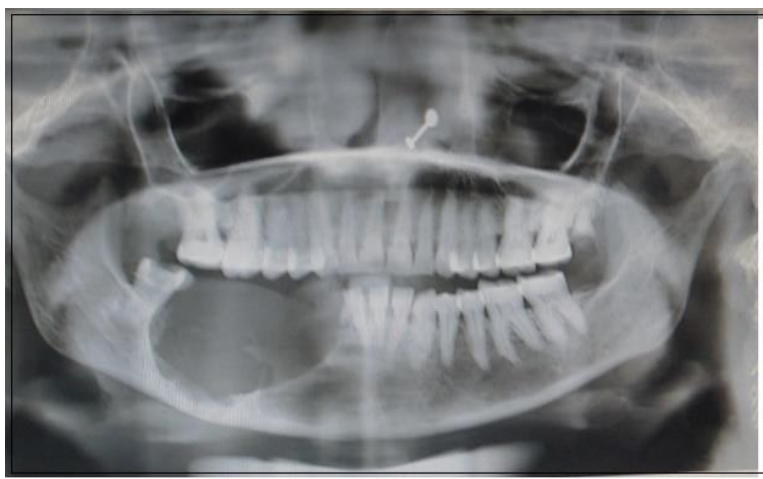

Fig. 2: OPG showing radiolucency in the posterior mandible 


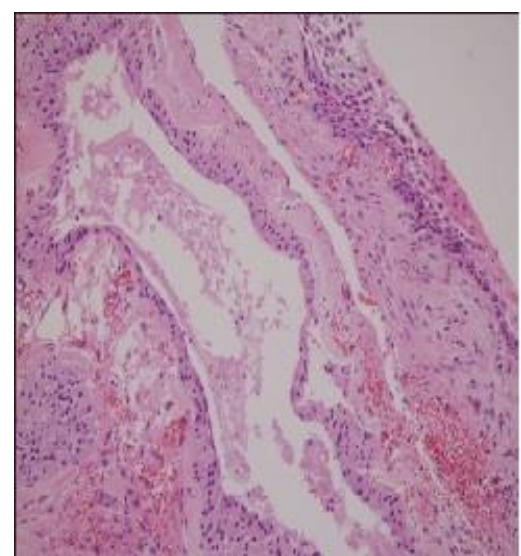

Fig. 3: Histology of unicystic ameloblastoma

\section{Discussion}

Unicystic ameloblastoma, a variant of ameloblastoma was first described by Robinson and Martinez. ${ }^{3}$ Unicystic ameloblastomas are most often seen in younger patients, with about $50 \%$ of tumors diagnosed during the second decade of life. ${ }^{4}$ Most of the males are affected than females. More than $90 \%$ of unicystic ameloblastomas are found in the mandible, usually in the posterior regions. The lesion is often asymptomatic, although large lesions may cause a painless swelling of the jaws. ${ }^{5}$ In many patients, this lesion typically appears as a circumscribed radiolucency that surrounds the crown of an unerupted mandibular third molar. Clinically resembling a dentigerous cyst. ${ }^{6}$ In some cases the radiolucent area has scalloped margins but is still a unicystic ameloblastoma. $^{7}$ There were many debates regarding whether unicystic ameloblastoma develops de novo or arises in an existing cyst. ${ }^{8}$ In the present situation it is seen in edentulous area of posterior region with molar $\left(3^{\text {rd }}\right.$ molar $)$ being distally tilted. Possibility of misdiagnosing such cases as dentigerous cyst poses a problem where repeat and deeper biopsies are advisable to reveal the underlying tumorous structure. However the biological behaviour has to be seen as this shows quick growth. Histologically it is of 3 types namely, luminal, intraluminal, mural. These tumors are usually treated by enucleation. Recurrence rates of $10 \%$ to $20 \%$ were seen after enucleation and curettage of unicystic ameloblastoma. ${ }^{9}$ This range is considerably less than the $50 \%$ to $90 \%$ recurrence rates noted after curettage of conventional solid and multicystic intraosseous ameloblastomas. ${ }^{10}$

\section{Conclusion}

The diagnosis of unicystic ameloblastoma is mostly based on the clinical, radiological and histological findings. The ability to predict helps to cure the disease in proper way. The pathologist also should be able to differencite this with other amelobastomas. It has to properly differenciated from dentigerous cyst as it is mostly confused with dentigerous cyst.

\section{Source of funding}

None.

\section{Conflict of interest}

None.

\section{References}

1. Paikkatt VJ, Sreedharan S, Kanna VP. Unicystic ameloblastoma of the maxilla: A case report. J Indian Soc Pedod Prev Dent 2007;25:106-10.

2. Li TJ, Wu YT, Yu SF, Yu GY. Unicystic ameloblastoma: A clinicopathologic study of 33 Chinese patients. Am J Surg Pathol 2000;24:1385-92.

3. Robinson L, Martinez MG. Unicystic ameloblastoma: A prognostically distinct entity. Cancer 1977;40:2278-85.

4. Gardner DG, Corio RL. Plexiform unicystic ameloblastoma: A variant of ameloblastoma with a low recurrence rate after enucleation. Cancer 1984;53:1730-5.

5. Neville, Brad W. 2009. Oral and maxillofacial pathology. St. Louis, Mo: Saunders/Elsevier.

6. Reconstr Scie 2004, 113(1):80-87. 5. Philipsen HP, Reichart PA: Unicystic ameloblastoma. Odontogenic tumors and allied lesions London: Quintessence Pub. Co. Ltd 2004, 77-86.

7. i TJ, Kitano M, Arimura K, Sugihara K. Recurrence of unicystic ameloblatoma: A case report and review of the literature. Arch Pathol Lab Med 1998;122:371-4.

8. Ackermann GL, Altini M, Shear M. The unicystic ameloblastoma: A clinicopathological study of 57 cases. J Oral Pathol 1988;17:541-6.

9. Thankappan S, Thomas V, Kandamparambil S, Nair S. Unicystic ameloblastoma: 3 case reports and review of literature. J Indian Acad Oral Med Radiol 2008;20:65-70.

10. Peter A. Reichart, PA, Phillipsen HP. Odontogenic tumors and allied lesions. Chicago: Quintessence Pub 2004. p. 77-86.

How to cite this article: Hassan SA, Bhateja S. Unicystic ameloblastoma- A case report. Int J Oral Health Dent 2019;5(3):145-6. 